Abstract

\title{
On Superstar-Generalized Statistical Regression ${ }^{\dagger}$
}

\section{Second Bwanakare}

University of Information Technology and Management, Rzeszow, Poland

+ Presented at the Entropy 2021: The Scientific Tool of the 21st Century, 5-7 May 2021; Available online: https://sciforum.net/conference/Entropy2021/.

Published: 5 May 2021

Recent academic literature has confirmed the existence of robust structures characterizing most of many real world systems described by the power law(PL). Intuitively, PL becomes more legible at the level of high frequency series fluctuations in their upper part (thus an asymptotic law), knowing that for certain phenomena the lower part could be generated by this same law. In phenomenological terms, fluctuations in the upper part of natural or social series generally lead to the criticality point at the eve of phase change (e.g., change in physical properties and behavior of matter at a certain temperature human eccentric behavior under the effect of higher emotion, financial crash, etc.). The present proposal goes beyond the traditional statistical methodology mainly based on the Central Limit Theorem(CLT) which presents serious limits when dealing with complex dynamic phenomena. In front of such endogenous methodological problem, a large community of statisticians instead try to find out new techniques fundamentally within the traditional CLT. In line with recent research, this paper recall and extends the fundaments of a recent approach of non-extensive cross-entropy econometrics (NCEE) (Bwanakare 2019). Equivalently, and to honor Rosen (see Rosen 1981, Gabaix 2009) who, for the first time, used the expression of "Economics of Superstars" to exemplify the presence of PL in the case of artistic earnings (stars inclusively), we propose here the expression of Superstars Generalized statistical regression (SGSR). Thus, this paper proposes a theoretical, power law-based simultaneous regression equation to be estimated trough the q-generalised Kullback-Leibler statistical theory of information.

(C) 2021 by the author. Licensee MDPI, Basel, Switzerland. This article is an open access article distributed under the terms and conditions of the Creative Commons Attribution (CC BY) license (http://creativecommons.org/licenses/by/4.0/). 